\title{
The Danish Centre for Strategic Research in Type 2 Diabetes (DD2) Project: rationale and planned nationwide studies of genetic predictors, physical exercise, and individualized pharmacological treatment
}

This article was published in the following Dove Press journal:

Clinical Epidemiology

20 September 2012

Number of times this article has been viewed

\author{
Charlotte Steffensen' \\ Reimar W Thomsen ${ }^{2}$ \\ Allan Vaag ${ }^{3}$ \\ Henning Beck-Nielsen ${ }^{4}$ \\ Jens Sandahl Christiansen' \\ Torben Hansen ${ }^{5,6}$ \\ Oluf Pedersen ${ }^{5,6}$ \\ Jørgen Rungby' \\ 'Aarhus University Hospital, \\ Department of Medicine, Aarhus, \\ ${ }^{2}$ Aarhus University Hospital, Institute \\ of Clinical Medicine, Department \\ of Clinical Epidemiology, Aarhus, \\ ${ }^{3}$ Copenhagen University Hospital \\ "Rigshospitalet", Department of \\ Endocrinology, Copenhagen, ${ }^{4}$ Odense \\ University Hospital, Department of \\ Endocrinology, Odense, ${ }^{5}$ The Novo \\ Nordisk Foundation Centre for Basic \\ Metabolic Research, Faculty of Health \\ Sciences, University of Copenhagen, \\ Copenhagen, ${ }^{6}$ Faculty of Health \\ Sciences, University of Aarhus, Aarhus, \\ Denmark
}

\begin{abstract}
Here we provide an overview of the rationale and methods of a series of planned population based studies within the Danish Centre for Strategic Research in Type 2 Diabetes (DD2) Project. The project aims to support and evaluate ongoing political and administrative efforts to implement nationwide guidelines for maintaining metabolic control in newly diagnosed type 2 diabetes (T2D) patients to prevent diabetic complications and improve quality of life. The DD2 is designed as a prospective cohort study (collection of epidemiological data) supplemented by randomized clinical intervention trials (on physical exercise and individualized pharmacological treatment) and the establishment of a biobank comprised of material from a large number of newly diagnosed T2D patients. Inclusion of the majority of newly diagnosed T2D patients as they are diagnosed at their general practitioner or diabetes hospital outpatient clinics and entered into the DD2 cohort will establish a nationwide database comprising a large number of future incident cases of T2D in Denmark. These cases will form the project cohort of the DD2. Within the first 6 months of diagnosis, all patients will be invited to contribute to a biobank of DNA, plasma, urine, and tissue sampling. The DNA biobank will enable future studies of the effect of pharmacological treatment and outcome in subsets of patients with specific genetic risk profiles covering disease etiology and specific drug kinetics and metabolism. We will also perform two clinical intervention trials examining: the effectiveness of physical exercise on diabetes-related outcomes and the impact of trial outcomes on individualized pharmacological treatment. Moreover, the DD2 will serve as a platform for testing and developing new antidiabetic drugs. All together, we expect this study to contribute to substantially improved diabetes care in T2D patients locally and abroad.
\end{abstract}

Keywords: type 2 diabetes, prognosis, intervention, physical exercise

\section{Background and rationale of the planned studies Genetic predictors}

The incidence of type 2 diabetes (T2D) is increasing. The disease often carries severe complications. Since 1992, several genetic subtypes of monogenetic diabetes have been described in which gene mutations result in diabetes primarily through beta-cell dysfunction. ${ }^{1-4}$ This new knowledge means that patients who were previously categorized clinically as having maturity-onset diabetes of the young (MODY), permanent neonatal diabetes mellitus, or transient neonatal diabetes mellitus, can now be classified 
by genetic subgrouping. Definition of the genetic subgroup can result in appropriate treatment, genetic counseling, and prognostic information. In contrast, until recently, progress in identifying the genetic variants influencing predisposition to polygenic and much more common forms of T2D has been slow. However, recent advances have begun to alter the situation. Well-powered candidate gene studies and a number of genome-wide association studies have extended the number of genetic loci to 20 harboring common variants that are implicated in diabetes susceptibility. Furthermore, a number of loci associated with obesity, dyslipidemia, hypertension, and cardiovascular disease have been identified. Some of these gene variants seem to offer new avenues for clinical translation. Recently, genetic variation was established to alter the response to therapy in T2D. Carriers of the T2D TCF7L2 rs7903146 T-allele showed a decreased response to sulfonylureas but not to metformin. ${ }^{5}$ Most of the recently identified variants seem to affect beta-cell function, ${ }^{6}$ but the fat mass and obesity-associated gene (FTO)-variants have been shown to influence T2D risk through a primary effect on weight and obesity. Interestingly, the impact of the FTO variants on risk of obesity and T2D seems to be influenced by level of physical exercise. Physical inactivity is associated with decreased insulin sensitivity and a body mass index increase of nearly $2 \mathrm{~kg} / \mathrm{m}^{2}$ in those carrying two risk variants, whereas no major effect of sedentary lifestyle were found among noncarriers of FTO risk variants. ${ }^{7,8}$ Thus, the identification of new genes and pathways responsible for T2D predisposition and increased risk of diabetic complications offers opportunities for developing novel therapeutic and preventive approaches. Furthermore, the identification of additional genetic variants, both protective and those increasing risk, may render it possible to use patterns of predisposition to tailor individual management of these conditions.

\section{Physical exercise}

One key intervention in the treatment of T2D is lifestyle change. In this respect, dietary interventions are based on solid scientific data ${ }^{9}$ but it is presumed that increased physical activity is also an important part of treatment in T2D patients. This is indicated by the recent report from The Danish Commission on Prevention (www.forebyggelseskommisionen.dk/files/filer/faktaark_motion.pdf) recommending exercise training by prescription to subjects at high risk including T2D patients. Although studies have shown that the onset of T2D may be postponed by around 2 years by physical exercise when implemented in the prediabetic stage (impaired glucose tolerance), ${ }^{9-11}$ it has never been documented that patients with overt T2D are able to increase their level of physical exercise over longer periods and it remains unknown if physical exercise training may improve quality of life, reduce diabetic complications, and prolong life expectancy when initiated in patients with overt T2D.

\section{Individualized pharmacological treatment}

One problem related to improvement in the outcome of T2D is that the algorithms for pharmacological treatment are not individualized but are based on cohort studies and applied to patients in general, independent of the fact that T2D is a very heterogeneous disease with different pathophysiological defects underlying hyperglycemia. Some patients recognized as T2D (about 10\%) suffer from latent autoimmune diabetes in adults (LADA), a disease destroying beta-cells, and others suffer from monogenetic MODY forms (about $1 \%-2 \%$ ). However, around $80 \%$ of T2D patients develop hyperglycemia due to multifactorial and multigenetic factors. ${ }^{12}$ Most - but not all - patients are insulin resistant, whereas in other patients the beta-cell defects are dominant. In those situations, different genetic defects may be involved so both the genotype and the phenotype must be characterized to apply the appropriate treatment and, as a minimum, the degree of insulin resistance, beta-cell capacity, degree of autoimmunity, gene variants, and the metabolic profile must be measured in newly diagnosed T2D patients to optimize the therapeutic intervention. In "conventional" evidencebased medicine, most data are generated from large clinical intervention trials treating all subjects in the same way eg, with a specific drug. ${ }^{13-17}$ However, it is often not recognized that even if the intervention group performed better than the control group, it does not prove conclusively that all subjects will benefit from the intervention; some may in fact be harmed. Nevertheless, we used these data to develop algorithms for treatment of all T2D patients as if they were a homogenous group. Thus, this study differs from studies such as ADVANCE ${ }^{18}$ and $\mathrm{ACCORD}^{19}$ in that it is not a randomized controlled trial but individualized algorithms based on specific characterization of the individual genotype. Phenotype will be tested prospectively in specific cohorts.

To prevent T2D and improve treatment of the metabolic abnormalities behind the disease, with the main aim of preventing complications, cost-effective national organization and intervention are needed. The individualized treatment described is costly, but it is our experience, based on the Funen model project, ${ }^{20}$ that correct treatment costs less in the long-term. Thus, although intervention against diabetic 
complications may be expensive, it will be cost-effective in the long-term since treatment of severe complications is even more costly. With this in mind, health economics will be an important part of the DD2 study.

\section{Design and methods of the planned studies}

The DD2 project will consist of two parts: a large populationbased prospective cohort study including the establishment of databases and a biobank that will allow a number of ensuing nested case-control studies and clinical intervention. The DD2 aims to include most newly diagnosed T2D patients from 2010 to 2015 - that is, about 50,000 patients. In the population-based cohort study, we will follow patients over time for diabetes outcomes including their use of medications, hospital contacts and diagnoses of diabetic complications, surgical procedures, dialysis, socioeconomic prognosis, and total and cause-specific mortality. We will examine predictors of metabolic results and clinical endpoints (late diabetic complications and mortality), including genetic predictors by using a new biobank of DNA. Also we will examine a subset of patients with specific genetic risk profiles covering disease, etiology and specific drug genetics as well as metabolism, including non-DNA biomarkers. In the clinical intervention part, we will test the hypothesis that physical exercise is an important part of treatment of T2D and investigate the importance of individualized pharmacological treatment and the cost-effectiveness of this kind of treatment. Finally, beside these projects, we aim to develop a platform for testing and developing new antidiabetic drugs. This part will be carried out in collaboration with Novo Nordisk, Copenhagen, Denmark.

\section{Establishing a nationwide cohort of future incident cases of T2D and following them in Danish registries}

To conduct this nationwide cohort study in Denmark, we will use the unique Danish registration system. The logistics of patient enrollment in the DD2 from general practitioners and hospital outpatient clinics have been described in detail by Nielsen et al. ${ }^{21}$ The Danish National Registry of Patients covers all hospitals serving the entire Danish population of 5.5 million people. The records include dates of hospital admission and discharge, type and date of surgical procedures performed since 1977, and up to 20 discharge diagnoses, classified according to the International Classification of Diseases, 8th revision (ICD-8) until December 31, 1993, and 10th revision thereafter (ICD-10) ${ }^{22}$. In all Danish registries, patients are identified by their civil registration numbers. These unique identifiers assigned at birth or at immigration are stored in the Danish civil registration system - a nationwide registry of all persons residing in Denmark since April 1, 1968 - along with birth date, residency status, and dates of immigration, emigration, and death (if any). The registration number identifies the patient in all health registries thus enabling linkage and complete follow up data achieved from a number of data sources. These include comorbidities from the National Registry of Patients and pharmacological treatment from the Danish Registry of Medical Products Statistics. Furthermore, it is possible to link with nationwide laboratory databases to ascertain the quality of diabetes care in Denmark, for example, to the The Danish Diabetes Database for Adults, ${ }^{20,21,23}$ as well as to other relevant health and demographic databases. The Danish registration system makes possible a comprehensive follow-up on clinical endpoints on each patient in the DD2 cohort. $^{24}$

\section{The Danish health care system and application of national diabetes guidelines}

The Danish health care system can be used free at the point of delivery by everybody who is a permanent resident in Denmark, as described in more detail elsewhere. ${ }^{21}$ This includes diabetes care. All permanent residents receive a national health insurance card that works as an identity card and must be presented at all visits to doctors, emergency rooms, and hospitals. The five regions of Denmark are responsible for providing hospital care and own and run their own hospitals, outpatient clinics, and prenatal care centers. The regions also finance general practitioners, specialists, physiotherapists, dentists, and pharmaceuticals. Self-employed general practitioners act as gatekeepers to secondary care; thus, all patients have to be referred to hospital or outpatients clinics by their general practitioner for specialized diagnosis, treatment, and care.

Vital to the DD2 project is the inclusion of a major proportion of new diabetes cases, as they are registered with this diagnosis by their general practitioner or at public hospitals. These cases will form the project database. The DD2 project aims to support ongoing political and administrative efforts to implement nationwide guidelines. The goal of these guidelines is to provide referral of all patients who are newly diagnosed with T2D to a hospital diabetes outpatient clinic for registration and participation in a diabetes education program. Application of such universal guidelines will minimize referral bias and reduce variability 
in the quality of initial primary care. Thus, one of the goals is the establishment of an efficient, centralized, and uniform registration of all incident cases, whereby risk values and clinical outcome can be followed continuously and used for instruction.

\section{Establishing a repository of biomaterials from patients with incident cases of T2D}

Within the first 6 months of diagnosis, all patients registered as incident cases will be invited to contribute to a DNA biobank. After signing a statement of consent, patients will be asked to provide a blood and urine sample at their general practitioner or at the diabetes outpatient clinic, which will be stored at the biobank, and to fill out a short questionnaire. The Danish registration system allows us to recover, at regular intervals, data on lipid profiles, blood pressure, glycemic control, and body composition of patients enrolled in the DD2 project, and these data sources will be used to follow patients over time. As additional data can be retrieved, patients need only visit an outpatient diabetes clinic or their general practitioner clinic once to be fully enrolled in the DD2 project. (For further information about implementations and logistics see Nielsen et al. $)^{21}$

Genomic DNA will be genotyped in the entire cohort, with a focus on known risk variants for T2D. If relevant, it will also be sequenced for the identification of new variants important for outcome. Variants proven associated with obesity, dyslipidemia, hypertension, and cardiovascular disease will be genotyped. We plan to test the effects of both individual gene variants and combinations of gene variants on drug response and the development of micro- and macrovascular complications. Further, we will examine whether the effects of early intensified physical exercise is modified by specific variants. For biomarkers other than DNA, after identification of incident cases (at 6-month intervals in The Danish National Registry of Patients), a centralized randomization procedure will identify half of the study population, who will then be invited to contribute to a bioregistry of blood samples. We will specifically measure fasting plasma C-peptide to monitor beta-cell function and glutamic acid decarboxylase antibodies to monitor autoimmunity, as well as other molecules related to insulin resistance, such as fasting

Table I Timescale for The Danish Centre for Strategic Research in Type 2 Diabetes (DD2) Project

\begin{tabular}{|c|c|c|c|c|c|c|c|c|c|c|c|c|c|c|c|c|c|}
\hline & \multirow{2}{*}{$\frac{\text { Year 20I I }}{\text { Jan }}$} & \multicolumn{12}{|c|}{ Year 2012} & \multicolumn{4}{|c|}{ Year 2013} \\
\hline & & Jan & Feb & Mar & Apr & May & Jun & Jul & Aug & Sep & Oct & Nov & Dec & Jan & Feb & Mar & Apr \\
\hline \multicolumn{18}{|l|}{ Event } \\
\hline \multicolumn{18}{|l|}{ Patient enrollment into DD2. } \\
\hline \multicolumn{18}{|l|}{ Registration of newly diagnosed } \\
\hline \multicolumn{18}{|l|}{ T2D subjects for the first year } \\
\hline \multicolumn{18}{|l|}{ should be finalized; samples } \\
\hline \multicolumn{18}{|l|}{ should be collected and stored } \\
\hline \multicolumn{18}{|l|}{ at either Vejle Hospital or the } \\
\hline \multicolumn{18}{|l|}{ National Institute for Health Data } \\
\hline \multicolumn{18}{|l|}{ and Disease Control; start of the } \\
\hline \multicolumn{18}{|l|}{ intervention studies. Methods for } \\
\hline \multicolumn{18}{|l|}{ recruitment established. } \\
\hline \multicolumn{18}{|l|}{ Registration of newly diagnosed } \\
\hline \multicolumn{18}{|l|}{ T2D subjects for the first year } \\
\hline \multicolumn{18}{|l|}{ should be finalized; samples should } \\
\hline \multicolumn{18}{|l|}{ be collected and stored at either } \\
\hline \multicolumn{18}{|l|}{ Vejle Hospital or National Institute } \\
\hline \multicolumn{18}{|l|}{ for Health Data and Disease } \\
\hline \multicolumn{18}{|l|}{ Control; start of the intervention } \\
\hline \multicolumn{18}{|l|}{ studies. Methods for recruitment } \\
\hline \multicolumn{18}{|l|}{ established. } \\
\hline \multicolumn{18}{|l|}{ Development of the second clinical } \\
\hline \multicolumn{18}{|l|}{ report; end of intervention studies } \\
\hline on individualized treatment. & & & & & & & & & & & & & & & & & \\
\hline Development of the third clinical & & & & & & & & & & & & & & & & & \\
\hline report; end of the physical & & & & & & & & & & & & & & & & & \\
\hline training trial & & & & & & & & & & & & & & & & & \\
\hline Reporting of main results from & & & & & & & & & & & & & & & & & \\
\hline the DD2 study. & & & & & & & & & & & & & & & & & \\
\hline
\end{tabular}


serum insulin. This material will allow analysis of proteins or small molecule biomarkers.

\section{Clinical intervention study: randomized clinical substudies}

Effects of an early physical exercise program

Lifestyle changes, such as dietary interventions and increased physical exercise, are proposed as important and one of the key interventions in the treatment of T2D. ${ }^{10,11}$ The Danish Commission on Prevention recommends the prescription of exercise training to subjects at high risk of or with T2D. In Denmark, the municipalities are responsible for population-directed preventative efforts to increase the level of physical exercise. We expect $40 \%$ of the municipalities in Denmark will volunteer to join this DD2 substudy; thus, we expect to include a minimum of $5000 \mathrm{~T} 2 \mathrm{D}$ patients over 2 or more years. All interested municipalities will be instructed in different methods to enhance the level of physical exercise. Ten representative municipalities will be selected to join an "interval walking" program. The remaining municipalities will serve as controls. After
5 years of intervention, we will investigate whether physical exercise has lowered the risk of cardiovascular disease among T2D patients.

\section{Individualized pharmacological treatment}

During the DD2, we will set up prospective studies in cohorts to test the importance of individually tailored treatment based on the pathophysiological characterization of each subject. In this respect, we wish to characterize T2D patients with autoimmunity - that is, glutamic acid decarboxylase antibodies - termed "LADA," and patients with specific MODY subtypes. These patients will be offered a specific treatment based on pathophysiological characterization. LADA patients will be randomized to either the general national algorithm or early insulin treatment. In the MODY group, treatment will be individualized based on genotype, since each genotype group has its own prognosis. The remaining DD2 patients in this case will also be treated in accordance with the general guidelines. In both the LADA and MODY intervention trials, the number of patients will be too small for clinical endpoint trials (some $5 \%-10 \%$ of

\begin{tabular}{|c|c|c|c|c|c|c|c|c|c|c|c|c|c|c|c|c|c|c|c|c|c|c|}
\hline \multirow[b]{2}{*}{ May } & \multirow[b]{2}{*}{ Jun } & \multirow[b]{2}{*}{ Jul } & \multirow[b]{2}{*}{ Aug } & \multirow[b]{2}{*}{ Sep } & \multirow[b]{2}{*}{ Oct } & \multirow[b]{2}{*}{ Nov } & \multirow[b]{2}{*}{ Dec } & \multicolumn{12}{|c|}{ Year 2014} & \multicolumn{3}{|c|}{ Year 2015} \\
\hline & & & & & & & & Jan & Feb & Mar & Apr & May & Jun & Jul & Aug & Sep & Oct & Nov & Dec & Jan & Feb & Mar \\
\hline
\end{tabular}


the diabetes population) and metabolic criteria will thus be used, for example, $\mathrm{HbA}_{1 \mathrm{c}}$, blood pressure, lipids, and urine albumin excretion. In the large group of non-LADA and non-MODY patients ( $80 \%$ of all T2D patients), two major phenotypes seem to be present (as defined by the World Health Organization); namely, patients with severe insulin resistance (defined by homeostasis model assessment estimates) and patients with normal insulin sensitivity, but severe defects in insulin secretion (here defined by the fasting plasma C-peptide concentration). Patients with severe insulin resistance ( $25 \%$ of the upper quartile) will be identified and randomized to treatment with insulin sensitizers, combined with dipeptidyl peptidase IV inhibitors or glucagon-like peptide- 1 analogs if needed, or to the general national guidelines standard treatment suggested by national guidelines. As we will also be involved with small groups, we will focus on metabolic outcome data, as previously stated. Patients suffering from severe beta-cell defects (ie, with fasting plasma C-peptide $<300 \mathrm{pmol} / \mathrm{L}$ ) will be randomized to insulin treatment or the general guideline algorithm and evaluated as described for the insulin-resistant subjects. Based on a power calculation, with each study running for 2 years, an expected reduction in $\mathrm{HbA} 1 \mathrm{c}$ of $0.4 \%$ requires more than 250 patients in each project arm. Testing our hypothesis of individualized treatment may enable us to develop a more adequate algorithm for the pharmaceutical treatment of T2D patients.

\section{Use of results and timescale}

Based on the updated results from the survey, the investigators will ensure immediate translation of the results into more individual clinical guidelines for the daily treatment and monitoring of T2D. The annual reports will include detailed health economics cost-benefit analyses and will be forwarded to the National Steering Committee of the Danish National Board of Health for implementation in new guidelines.

The timescale for the DD2 project is described in Table 1.

\section{Conclusion}

The overall aim of the project is to near-normalize metabolic control in newly diagnosed T2D patients to prevent complications and improve quality of life by continuously developing and implementing guidelines and organizational plans as results emerge from epidemiological and interventional substudies. Systematic follow-up will be performed continuously and through the participation of both scientific and regulatory bodies guidelines will evolve on all levels, individually and nationally.

\section{Acknowledgment}

DD2 is the acronym for 'The Danish Centre for Strategic Research in Type 2 Diabetes' supported by the Danish Agency for Science (grant no. 09-067009 and 09-075724). DD2 is also supported by The Danish Health and Medicines Authority, The Danish Diabetes Association and an unrestricted donation from Novo Nordisk A/S. The partners of the project are listed on the project website at www.DD2.nu.

\section{Disclosure}

The authors report no conflicts of interest in this work.

\section{References}

1. Fajans SS, Bell GI, Polonsky KS. Molecular mechanisms and clinical pathophysiology of maturity-onset diabetes of the young. $N$ Engl $J$. 2001;345:971-980.

2. Froguel P, Zouali H, Vionnet N, et al. Familial hyperglycemia due to mutations in glucokinase - definition of a subtype of diabetes. $N \mathrm{Engl}$ J Med. 1993;328:697-702.

3. Meur G, Simon A, Harun N, et al. Insulin gene mutations resulting in early-onset diabetes: marked differences in clinical presentation, metabolic status, and pathogenic effect through endoplasmic reticulum retention. Diabetes. 2010;59:653-661.

4. Murphy R, Ellard S, Hattersley AT. Clinical implications of a molecular genetic classification of monogenic beta-cell diabetes. Nat Clin Pract Endocrinol Metab. 2008;4:200-213.

5. Pearson ER, Donnelly LA, Kimber C, et al. Variation in TCF7L2 influences therapeutic response to sulfonylureas: a GoDARTs study. Diabetes. 2007;56:2178-2182.

6. Grarup N, Sparsø T, Hansen T. Physiologic characterization of type 2 diabetes-related loci. Curr Diab Rep. 2010;10(6):485-497.

7. Andreasen CH, Stender-Petersen KL, Mogensen MS, et al. Low physical activity accentuates the effect of the FTO rs 9939609 polymorphism on body fat accumulation. Diabetes. 2008;57(1):95-101.

8. Hertel JK, Johansson S, Sonestedt E, et al. FTO, type 2 diabetes, and weight gain throughout adult life: a meta-analysis of 41,504 subjects from the Scandinavian HUNT, MDC, and MPP studies. Diabetes. 2011;60:1637-1644.

9. Gæde P, Lund-Andersen H, Parving HH, Pedersen O. Effect of a multifactorial intervention on mortality in type 2 diabetes. New Engl J Med. 2008;358:580-591.

10. Byberg L, Melhus H, Gedeborg R, et al. Total mortality after changes in leisure time physical activity in 50 year old men: 35 year follow-up of population based cohort. BMJ. 2009;338:b688.

11. Tuomilehto J, Lindström J, Eriksson JG, et al. Prevention of type 2 diabetes mellitus by changes in lifestyle among subjects with impaired glucose tolerance. $N$ Engl J. 2001;344:1343-1350.

12. Gerich JE. The genetic basis of type 2 diabetes mellitus: impaired insulin secretion versus impaired insulin sensitivity. Endocr Rev. 1998;19: 491-503.

13. ADVANCE Collaborative Group. Patel A, MacMahon S, et al. Intensive blood glucose control and vascular outcomes in patients with type 2 diabetes. N Engl J Med. 2008;358:2560-2572.

14. Action to Control Cardiovascular Risk in Diabetes Study Group.Gerstein $\mathrm{HC}$, Miller ME, et al. Effects of intensive glucose lowering in type 2 diabetes. $N$ Engl J Med. 2008;358:2545-2559.

15. Holman RR, Paul SK, Bethel MA, Matthews DR, Neil HA. 10-year follow-up of intensive glucose control in type 2 diabetes. NEngl J Med. 2008;359:1577-1589.

16. Home PD, Pocock SJ, Beck-Nielsen H, et al. Rosiglitazone evaluated for cardiovascular outcomes - an interim analysis. $N$ Engl J Med. 2007;357(1):28-38. 
17. Poulsen MK, Henriksen JE, Hother-Nielsen O, Beck-Nielsen H. The combined effect of triple therapy with rosiglitazone, metformin, and insulin aspart in type 2 diabetic patients. Diabetes Care. 2003;26: 3273-3279.

18. Action in Diabetes and Vascular Disease: Preterax and Diamicron mr Controlled Evaluation. (ADVANCE). Available from: http://www. advance-trial.com.

19. Action to Control Cardiovascular Risk in Diabetes (ACCORD). Available from: http://www.accordtrial.org.

20. The Funen Telemedicine Initiative 2004-2006. Telemedicine in Practical Application. Odense: Danish Centre for Health Telematics; 2006. Available from: http://www.medcom.dk/dwn1177. Accessed March 30, 2012.

21. Nielsen JS, Steffensen C, Thomsen RW, Christiansen JS. The Danish Centre for Strategic Research in Type 2 Diabetes (DD2): implementation and logistics. Clin Epidemiol. 2012;4(Supp 1). In press.
22 World Health Organization, International Classification of Diseases (ICD). Available at www.who.int/classifications/icd/en/.

23. Thomsen RW, Friborg S, Nielsen JS, Schroll H, Johnsen SP. The Danish Centre for Strategic Research in Type 2 Diabetes (DD2): organization of diabetes care in Denmark and supplementary data sources for data collection among DD2 study participants. Clin Epidemiol. 2012;4(Supp 1). In press.

24. Thomsen RW, Nielsen JS, Ulrichsen SP, Pedersen L, Sigsgaard HansenAM, Nilsson T. The Danish Centre for Strategic Research in type 2 Diabetes (DD2) study: collection of baseline data from the first 580 patients. Clin Epidemiol. 2012;4(Supp1). In press.
Clinical Epidemiology

\section{Publish your work in this journal}

Clinical Epidemiology is an international, peer-reviewed, open access journal focusing on disease and drug epidemiology, identification of risk factors and screening procedures to develop optimal preventative initiatives and programs. Specific topics include: diagnosis, prognosis, treatment, screening, prevention, risk factor modification, systematic

\footnotetext{
Submit your manuscript here: http://www.dovepress.com/clinical-epidemiology-journal
}

\section{Dovepress}

reviews, risk \& safety of medical interventions, epidemiology \& biostatical methods, evaluation of guidelines, translational medicine, health policies \& economic evaluations. The manuscript management system is completely online and includes a very quick and fair peer-review system, which is all easy to use. 